\title{
Full genome sequence analysis of a 1-7-4-like PRRSV strain in Fujian Province, China
}

\author{
Jiankui Liu ${ }^{\text {Corresp., }, 2,}$, Chunhua Wei ${ }^{\text {Corresp., 1, }}{ }^{\text {, }}$ Zhifeng Lin ${ }^{3}$, Wei Xia ${ }^{1,2}$, Ying Ma ${ }^{1,2}$, Ailing Dai ${ }^{1,2}$, Xiaoyan Yang ${ }^{1,2}$ \\ ${ }^{1}$ College of Life Sciences, Longyan University, Longyan, China \\ ${ }^{2}$ Fujian Provincial Key Laboratory for the Prevention and Control of Animal Infectious Diseases and Biotechnology, Longyan University, Longyan, China \\ ${ }^{3}$ College of Animal Science,, Fujian Agriculture and Forestry University, Fuzhou, China \\ Corresponding Authors: Jiankui Liu, Chunhua Wei \\ Email address: liujiankui@lyun.edu.cn, weichunhua02@163.com
}

PRRS virus (PRRSV) has undergone rapid evolution and resulted in immense economic losses in worldwide. In the present study, a PRRSV strain named FJ0908 causing high abortion rate (25\%) and mortality (40\%) was detected in a swine herd in China. To determine if a new PRRSV genotype had emerged, we characterized the genetic characteristics of FJ0908. Phylogenetic analysis indicated that FJ0908 was related to 1-7-4like strains circulating in United States since 2014. Furthermore, the ORF5 sequence restriction fragment length polymorphism (RFLP) pattern of FJ0908 was 1-7-4. Additionally, FJ0908 had a 100 aa deletion (aa329-428) within nsp2, as compared to VR-2332, and the deletion pattern was consistent with most of 1-7-4 PRRSVs. Collectively, the data of this study contribute to the understanding of 1-7-4-like PRRSV molecular epidemiology in China. 


\section{Full genome sequence analysis of a 1-7-4-like PRRSV strain}

\section{2 in Fujian Province, China}

3 Jiankui Liu ${ }^{1,2 *}$, Chunhua Wei ${ }^{1,2 *}$, Zhifeng Lin $^{3}$, Wei Xia ${ }^{1,2}$, Ying Ma ${ }^{1,2}$, AiLing Dai, Xiaoyan

4 Yang ${ }^{1,2}$

5

6

7

8

9

10

1 College of Life Sciences of Longyan University, Longyan, Fujian Province, 364000, China

2 Fujian Provincial Key Laboratory for the Prevention and Control of Animal Infectious Diseases and

Biotechnology, Fujian Province, 364000, China

14

3 College of Animal Science, Fujian Agriculture and Forestry University, Fuzhou, Fujian Province, 5002, China

*Corresponding authors:

Dr. Jian-kui Liu; Dr. Chunhua Wei

College of Life Sciences of Longyan University, No.1, Dongxiao Road, Xinluo District, Longyan, 364000,

China

Tel: +86-597-2797255; Fax: +86-597-2793889

E-mail: liujiankui99@126.com; liujiankui@lyun.edu.cn; weichunhua02@163.com 


\section{ABSTRACT}

30

31

PRRS virus (PRRSV) has undergone rapid evolution and resulted in immense economic losses in worldwide. In the present study, a PRRSV strain named FJ0908 causing high abortion rate (25\%) and mortality (40\%) was detected in a swine herd in China. To determine if a new PRRSV genotype had emerged, we characterized the genetic characteristics of FJ0908. Phylogenetic analysis indicated that FJ0908 was related to 1-7-4-like strains circulating in United States since 2014. Furthermore, the ORF5 sequence restriction fragment length polymorphism (RFLP) pattern of FJ0908 was 1-7-4. Additionally, FJ0908 had a 100 aa deletion (aa329-428) within nsp2, as compared to VR-2332, and the deletion pattern was consistent with most of 1-7-4 PRRSVs. Collectively, the data of this study contribute to the understanding of 1-7-4-like PRRSV molecular epidemiology in China. 
INTRODUCTION

54

55

56

Porcine reproductive and respiratory syndrome (PRRS) is a global viral swine disease and causing severe economic loss in the global pig industry (Neumann et al., 2005; Zhou and Yang 2010; Holtkamp et al., 2013; Gao et al., 2017). PRRS virus (PRRSV), the causative agent of PRRS, is a small enveloped virus with positive-sense single-stranded RNA virus belonging to the Arteriviridae family in the Nidovirales order (Benfield et al., 1992; Meulenberg et al., 2000).

The PRRSV genome is about $15 \mathrm{~kb}$ in length and contains ten open reading frames (ORFs), ORF1a, 1b, 2a, 2b, 3, 4, 5a, 5, 6 and 7. ORF1a and ORF1b encode at least 16 non-structural proteins (nsps) (Nsp1 $\alpha$, Nsp1 $\beta$, Nsp2-6, Nsp2TF, Nsp2N, Nsp7a, Nsp7b and Nsp8-12), while ORF2-ORF7 encode viral structural proteins: GP2a, E (2b), GP3, GP4, GP5a, GP5, M, and N (Meulenberg et al., 2000; Wu et al., 2005; Firth et al., 2010; Fang and Snijder 2010; Fang et al., 2012).

PRRSV is characterized by extensive genetic variation. Based on global PRRSV classification systems, type 2 PRRSV strains in China can be classified into lineage 1 (NADC30-like), lineage 3 (QYYZ-like), lineage 5.1 (VR2332-like), and lineage 8.7 (JXA1-like) (Gao et al., 2017; Liu et al., 2017b; Guo et al., 2018). Fujian Province, one of the largest livestock trading areas in China, herd movements across provinces and national borders are also common. Hence, multiple PRRSV types co-exist in swine herds. Recently, PRRSV isolates of the ORF5 restriction fragment length polymorphism (RFLP) 1-7-4 viruses emerged in America, causing dramatic abortion "storms" in the sow herd (Alkhamis et al., 2016; van Geelen et al., 2018). Here, we report a genetic and phylogenetic analysis of PRRSV isolate FJ0908 belong to the ORF5 1-7-4 viruses in Fujian Province, China.

\section{MATERIALS AND METHODS}

\section{Clinical case}


77

78

79

80

81

82

83

84

85

86

87

88

89

90

91

92

93

94

95

96

97

98

99

100

101

102

In September 2018, severe reproductive and respiratory disease was observed in piglets in a farm.

The affected pigs had respiratory distress, and high abortion rate (25\%) and mortality (40\%).

\section{Strain identification and nucleotide sequencing}

PRRSV infection was confirmed by Real-time RT-PCR testing of the serum of affected pigs according to the manufacturer's instructions. The ORF5 sequence RFLP pattern was inferred according to Wesley et al. The complete genomic sequences of FJ0908 were amplified as described previously (Zhang et al., 2018). The PCR products were purified and cloned into pGEM-T Easy according to the manufacturer's instructions (Promega, Madison, WI, USA) and three recombinant clones of every fragment were sequenced by Ruibo Life Technologies Corporation (Beijing, China).

\section{Complete genomic sequence analysis}

Fifty-four representative type 2PRRSV sequences in GenBank were utilized in phylogenetic analyses (Table 1). Multiplex sequence alignments were performed using CLUSTAL X (version 1.83) and the phylogenetic relationships were assessed by MEGA 6.0 as described (Liu et al., 2017b). The ORF5 sequences were classified according to the global PRRSV classification systems (Shi et al., 2010).

Recombination events were detected using Simplot v 3.5.1 and the boot scanning analysis was performed with a 200-bp window, sliding along the genome alignments with a step size of 20 bp.

\section{RESULTS}

\section{Complete genomic sequence analysis}

The genomes of FJ0908 (GenBank accession no. MK202794) was $15112 \mathrm{nt}$ in length, excluding the poly (A) tail at the 3 'end, $300 \mathrm{nt}$ shorter than the genome of the prototypic VR2332. Genome alignments revealed that FJ0908 shared 83.6\% identity with JXA1, 84.7\% with VR2332, 82.2 \% with QYYZ, 86.3\% with NADC30, 97.3-97.6\% with 1-7-4 PRRSV family (IA/2014/NADC34, IA/2015/NADC35) and 98.7\% with LNWK130 (Table 2). 
103

104

105

106

107

108

109

110

111

112

113

114

115

116

117

118

119

120

121

122

123

124

125

126

127

128

129

The results also showed that 5'-UTR, ORF1a, ORF1b, ORFs 2-7 and the 3'-UTR of FJ0908 shared 97.2-98.2\% nucleotide homology with 1-7-4 PRRSV family (IA/2014/NADC34, IA/2015/NADC35), which was higher than the homology shared with other representative strains, indicating that FJ0908 strain belonged to 1-7-4 PRRSV. ORF1a and ORF1b encode 16 nsps of PRRSV, Nsp1 $\beta$ and Nsp2 are the most variable protein products among these nsps (Table 2). ORFs 2 to 7 encode the PRRSV structural proteins, among these structural proteins, GP2, GP3, GP4, GP5a and GP5 exhibited the most variance (Table 2).

\section{Amino acid analysis of Nsp2}

Nsp2 contains different deletions and insertions, as compared to VR2332 and is the most variable protein in PRRSV genome (Fang et al., 2011; Li et al., 2011). Strikingly, the nsp2 gene of the FJ0908 was 2640 nt in length and encoded 880 aa, with a 100 aa deletion (aa329-428) within nsp2, as compared to VR2332, and the deletion pattern was consistent with most of 1-7-4 PRRSVs.

\section{Antigenic analysis of GP2-GP5}

The antigenic regions (ARs) and glycosylation sites within the GP2, GP3, GP4, and GP5 proteins of FJ0908 were predicted and compared to OH/2014/ISU-7 IA/2014/ISU-8, IA/2014/NADC34, IA/2015/NADC35, IA/2015/NADC36, LNWK96, LNWK130, NV-21, NV-25, VR2332, CH-1a, JXA1 and NADC30.

In GP2, two antigenic regions (AR 41-55 and AR123-135) were confirmed in type 2 PRRSV (de Lima et al., 2006). The predicted AR at aa 41-55 were highly conserved and no aa substitution was detected in AR123-135. In GP3, four predicted antigenic regions (AR32-46, AR 51-105, AR 111-125, and AR 137-159) were proven (de Lima et al., 2006; Zhou et al., 2006; Wang et al., 2014). The AR comprising aa32-46, aa51-105, aa111-125, and aa137-159 of FJ0908 was most similar to 1-7-4 representative PRRSVs including LNWK130, but differed from VR2332, CH-1a, JXA1 and NADC30. GP4 has one predicted AR at aa51-65 (de Lima et al., 2006). FJ0908 had 1-2 aa substitutions as compared to the 1-7-4 representative PRRSVs, but had 2-6 aa substitutions as compared to VR2332, CH-1a, JXA1 and NADC30 and LNWK96. Additionally, no aa substitution was detected in AR51-65 between FJ0908 and LNWK130. The putative glycosylation sites in 
130

131

132

133

134

135

136

137

138

139

140

141

142

143

144

GP2-4 were completely conserved among the investigated strains, except for GP2 of isolate IA/2015/NADC36 that had one substitution $\mathrm{N}^{184} \mathrm{D}$.

GP5 is the major envelope protein encoded by ORF5 and is the most variable PRRSV protein. Sequences alignments of GP5 revealed that FJ0908 shares 87.0\%, 86.0-86.5\%, 85.5\%, 88.0-90.5\% and 97.5\% amino acid identity with VR-2332-like (VR2332 and BJ-4), JXA1-like (JXA1 and HuN4), QYYZ-like (FJFS and QYYZ), NADC30-like strains (NADC30 and ISU30), and 1-7-4like (IA/2014/NADC34, IA/2015/NADC35), respectively (Table 2). Furthermore, the restriction sites analysis showed that the ORF5 RFLP of FJ0908 has the same 1-7-4 pattern [1 (MluI $=0$ sites), $7($ HindII $=$ nt 88, 219, 360), $4($ SacII $=$ nt 24, 555) $]($ Figure S1).

GP5 has six ARs (AR1-15, AR27-35, AR37-51, AR149-156, AR166-181, and AR192200) (de Lima et al., 2006; Zhouet al., 2009). In GP5, the N-terminus ARs (AR1-15 and AR27$35)$ were very variable among all strains and only three aa substitutions $\left(K^{4} N, Q^{13} R\right.$ and $\left.L^{15} P\right)$ were found in the two antigenic regions between FJ0908 and LNWK130, whereas the other four ARs (AR 37-51, AR 149-156, AR 166-181, and AR 192-200) were conserved. GP5 had differential predicted N-glycosylation among PRRSV strains. FJ0908 possessed five predicted sites (N32, N44, N51, N57 and N59). Although N-glycosylation at N57 was not a novel finding, N57 was detected in most of the 1-7-4 sequences. The results also revealed that FJ0908 had the same Nglycosylation pattern as LNWK96 and LNWK130.

\section{Phylogenetic analysis}

PRRSV ORF5 is the most variable and has been used as a marker of PRRSV genetic variability. Based on global PRRSV classification systems, type 2 PRRSV was divided into nine monophyletic lineages (1-9) and lineage1 was further classified into 9 sublineages (1.1-1.9) (Shi et al., 2010; Guo et al., 2017). The ORF5-based phylogenetic tree showed that FJ0908, as well as 1-7-4 isolates including LNWK130, were clustered in sublineage 1.5 (Fig. 1A). Whole genome phylogenetic analysis also indicated that FJ0908 was most closely related to a genetic cluster in1-7-4-like lineage 1 (Fig. 1B).

\section{Recombination analysis}


157 To test for possible recombinant events within FJ0908 strain, we performed recombinant detection

158

159

160

161

162

163

164

165

166

167

168

169

170

171

172

173

174

175

176

177

178

179

180

181

182

183

using SimPlot v3.5.1 software. From the similarity plot, two recombination breakpoints within the FJ0908 genome were identified, which were located in Nsp1 (nt 760 and nt 1300) (Fig. 2A). To further confirm the putative recombination events, phylogenetic trees for each of the sequence regions identified during the analysis were generated, we identified two recombination breakpoints located in nsp1 (nt 760 and nt 1300) (Fig. 2B, Fig. 2C). The two breakpoints separated the genome of FJ0908 into 3 regions. For FJ0908, the region between the breakpoints (nt 760-1300) is closely related to ISU30 strain, the two regions between the breakpoints (nt 1-759 and nt 1301-15534) are closely related to IA/2014/NADC34. Collectively, the above results suggested that FJ0908 derived from recombination between IA/2014/NADC34 and ISU30 (Fig. 2). Moreover, LNWK130 strain (1-7-4-like PRRSV) firstly identified in Liaoning Province, China was also deriving from the recombination of 1-7-4 isolates and ISU30 (Zhang et al., 2018). However, the recombination pattern was different between LNWK130 and FJ0908. For LNWK130, the two breakpoints (nt 760-1300 and nt 1-759) are closely related to ISU30 strain, one breakpoints (nt 1301-15534) is closely related to IA/2014/NADC34. Additionally, sequences alignments of the recombination region (nt 1-759) revealed that FJ0908 shares $98.8 \%, 94.8 \%$ and $92.6 \%$ nucleotide identity with IA/2014/NADC34, ISU30 and NADC30, respectively, in contrast, LNWK130 shares 95.8\%, 95.9\% and 93.1\% nucleotide identity with IA/2014/NADC34, ISU30, and NADC30, respectively. Sequences alignments of the recombination region (nt 760-1300) revealed that FJ0908 shares 92.8\%, 94.3\% and 82.0\% nucleotide identity with IA/2014/NADC34, ISU30 and NADC30, respectively, similarly, LNWK130 shares $89.4 \%, 93.0 \%$ and $80.2 \%$ nucleotide identity with IA/2014/NADC34, ISU30, and NADC30, respectively. For all of the nsp sequences and structural proteins, the most variable regions were found in nsp1 $\beta$, nsp3, nsp5, nsp6, nsp8, GP3 and GP5 between FJ0908 and LNWK130.

\section{DISCUSSION}

PRRSV causes major economic losses in swine industry since 1990s. Notably, PRRSV continues to expand its genetic diversity. According to Shi et al., 2010, type 2 PRRSV was classified into 
184

185

186

187

188

189

190

191

192

193

194

195

196

197

198

199

200

201

202

203

204

205

206

207

208

209

210

nine monophyletic lineages based on ORF5 and extensive genetic variation exists among strains within each lineage. It is hard to define the PRRSV homologous, heterologous virus and pathogenic biotype only focused on single gene analysis. To classify and infer the likely pathogenic biotype, RFLP patterns of ORF5 for type 2 strains is standard approach for veterinarians (Wesley et al., 1998). The RFLP pattern 1-7-4 emerged in the US and has become prevalent since 2014, this nomenclature has been associated with severe disease in herds leading to significant economic losses (van Geelen et al., 2018). In the present study, FJ0908 was isolated in a farm with high abortion rate and mortality in sows, the restriction sites analysis revealed that the ORF5 RFLP of FJ0908 has the 1-7-4 pattern.

Comparison to PRRS sequences in GenBank indicated FJ0908 belonged to 1-7-4-like PRRSV. The genomic regions with the highest variation were found in Nsp1 $\beta$, Nsp2, ORF2, ORF3, ORF4, ORF5a and ORF5, the lowest variation were found in Nsp1 $\alpha$, Nsp8-12, and ORF6 (Table 2). FJ0908 had 100aa deletions within Nsp2 (corresponding to position 328-427 of VR2332 nsp2), as compared to the reference strain VR2332, and the deletion pattern was consistent with 1-7-4 viruses.

The pathogenesis of PRRSV has been linked to the N-glycosylation motifs at certain sites of GP2-GP5 by acting as a glycan shield against minimizing the viral neutralizing antibody response (Wissink et al., 2004; Ansari et al., 2006; Faaberg et al., 2006; Das et al., 2011; Delisle et al., 2012; Wei et al., 2012). Many reports have also suggested that N-glycosylation motifs in GP5 of PRRSV is important for viral infectivity and viral immune evasion (Wissink et al., 2004; Ansari et al., 2006; Jianget al., 2007; Delisle et al., 2012; Wei et al., 2012). In the present study, GP5 contained five predicted N-glycan motifs in FJ0908: N32, N44, N51, N57 and N59. More interestingly, the Chinese strains LNWK96, LNWK130 and FJ0908 have an additional N-glycan at 59 compared to 1-7-4 isolates in the United States.

Recombination may play an important mechanism in generating genetic diversity in PRRSV (Liu et al., 2011; Murtaugh et al., 2010). Most of the 1-7-4 PRRSV isolates may be most potentially derived from different recombination patterns occurring among the local strains in the 
211 United States (van Geelen et al., 2018). Recently, 1-7-4-like PRRSV strains, LNWK130 isolated

212 in Liaoning Province, China was reported to originate from recombination events between

213 IA/2014/NADC34 and ISU30. Currently, recombination events involving NADC30-like PRRSV

214 strains and other PRRSV strains frequently occurred in China (Zhao et al., 2015; Zhang et al.,

215 2016; Bian et al., 2017; Liu et al., 2017a; Liu et al., 2017c; Zhao et al., 2017; Wang et al., 2018;

216 Zhou et al., 2018; Liu et al., 2019). To test for possible recombinant events within FJ0908 strain,

217 we performed recombinant detection using SimPlot v3.5.1 software. Recombination analysis

218 performed with the available full-length genome sequences revealed FJ0908 maybe originate from

219 recombination events between IA/2014/NADC34 and ISU30. Although FJ0908 and LNWK130

220 maybe drive from recombination events between IA/2014/NADC34 and ISU30, the recombination

221 pattern of two strains were different. Two recombination breakpoints were identified in nsp1 (nt

222760 and nt 1300) in FJ0908 strain, whereas one recombination breakpoint in nsp2 (nt 1480) in

223 LNWK130, suggesting the ancestor of FJ0908 and LNWK130 were most probably transported

224 from different region of United States.

225 In conclusion, 1-7-4-like PRRSV was also detected in Fujian Province of China besides

226 Liaoning Province. Therefore, effective strategy should be taken to control 1-7-4-like PRRSV and

227 to monitor herd movements.

\section{CONCLUSION}

229 In summary, we thoroughly analyzed a new sublineage of PRRSV strain FJ0908 isolated 230 from Fujian Province, China on the basis of a comprehensive study with the full-length genome.

231 These novel sublineage 1.5 virus is closely related to the ORF5 RFLP 1-7-4 strains. Phylogenetic 232 and molecular evolutionary analyses indicated that FJ0908 originated from a natural 233 recombination event between IA/2014/NADC34 and ISU30. Our data enhance our understanding 234 of the PRRSV evolution in China.

\section{Data Availability}

236 The following information was supplied regarding data availability.

\section{REFERENCE}


238

239

240

241

242

243

244

245

246

247

248

249

250

251

252

253

254

255

256

257

258

259

260

261

262

263

Alkhamis MA, Perez AM, Murtaugh MP, Wang X, Morrison RB. 2016. Applications of Bayesian Phylodynamic Methods in a Recent U.S. Porcine Reproductive and Respiratory Syndrome Virus Outbreak. Frontiers in Microbiology 7: 67.

Ansari IH, Kwon B, Osorio FA, Pattnaik AK. 2006. Influence of N-linked glycosylation of porcine reproductive and respiratory syndrome virus GP5 on virus infectivity, antigenicity, and ability to induce neutralizing antibodies. Journal of Virology 80: 3994-4004.

Benfield DA, Nelson E, Collins JE, Harris L, Goyal SM, Robison D, Christianson WT, Morrison RB, Gorcyca D, Chladek D. 1992. Characterization of swine infertility and respiratory syndrome (SIRS) virus (isolate ATCC VR- 2332). Journal of Veterinary Diagnostic Investigation 4: 127-133.

Bian T, Sun Y, Hao M, Zhou L, Ge X, Guo X, Han J, Yang H. 2017. A recombinant type 2 porcine reproductive and respiratory syndrome virus between NADC30-like and a MLV-like: genetic characterization and pathogenicity for piglets. Infection Genetics and Evolution 54: 279-286.

Das PB, Vu HL, Dinh PX, Cooney JL, Kwon B, Osorio FA, Pattnaik AK. 2011. Glycosylation of minor envelope glycoproteins of porcine reproductive and respiratory syndrome virus in infectious virus recovery, receptor interaction, and immune response. Virology 410: 385-394.

de Lima M, Pattnaik AK, Flores EF, Osorio FA. 2006. Serologic marker candidates identified among B-cell linear epitopes of nsp2 and structural proteins of a North American strain of porcine reproductive and respiratory syndrome virus. Virology 353: 410-421.

Delisle B, Gagnon CA, Lambert ME, D'Allaire S. 2012. Porcine reproductive and respiratory syndrome virus diversity of Eastern Canada swine herds in a large sequence dataset reveals two hypervariable regions under positive selection. Infection Genetics and Evolution 12: $1111-1119$.

Faaberg KS, Hocker JD, Erdman MM, Harris DL, Nelson EA, Torremorell M, Plagemann PG. 2006. Neutralizing antibody responses of pigs infected with natural GP5 N-glycan 
264

265

266

267

268

269

270

271

272

273

274

275

276

277

278

279

280

281

282

283

284

285

286

mutants of porcine reproductive and respiratory syndrome virus. Viral Immunology 19: 294304.

Fang Y, Snijder EJ. 2010. The PRRSV replicase: exploring the multifunctionality of an intriguing set of nonstructural proteins. Virus Resarch 154: 61-76.

Fang Y, Treffers EE, Li Y, Tas A, Sun Z, van der Meer Y, de Ru AH, van Veelen PA, Atkins JF, Snijder EJ, Firth AE. 2012. Efficient-2 frameshifting by mammalian ribosomes to synthesize an additional arterivirus protein. Proceedings of the National Academy of Sciences of the United States of America 109: E2920-E2928.

Firth AE, Zevenhoven-Dobbe JC, Wills NM, Go YY, Balasuriya UB, Atkins JF, Snijder EJ, Posthuma CC. 2011. Discovery of a small arterivirus gene that overlaps the GP5 coding sequence and is important for virus production. Journal of General Virology 92: 1097-1106.

Gao JC, Xiong JY, Ye C, Chang XB, Guo JC, Jiang CG, Zhang GH, Tian ZJ, Cai XH, Tong GZ, An TQ. 2017. Genotypic and geographical distribution of porcine reproductive and respiratory syndrome viruses in mainland China in 1996-2016. Veterinary Microbiology 208: $164-172$.

Guo Z, Chen XX, Li R, Qiao S, Zhang G. 2018. The prevalent status and genetic diversity of porcine reproductive and respiratory syndrome virus in China: a molecular epidemiological perspective. Virology Journal 15(1): 2.

Holtkamp DJ, Kliebenstein JB, Neumann EJ, Zimmerman JJ, Rotto HF, Yoder TK, Wang C, Yeske PE, Mowrer CL, Haley CA. 2013. Assessment of the economic impact of porcine reproductive and respiratory syndrome virus on United States pork producers. Journal of Swine Health and Production 21: 72-84.

Jiang WM, Jiang P, Wang XL, Li YF, Wang XW, Du, Y. 2007. Influence of porcine 
287

288

289

290

291

292

293

294

295

296

297

298

299

300

301

302

303

304

305

306

307

308

309

310

311

312

reproductive and respiratory syndrome virus GP5 glycoprotein N linked glycans on immune responses in mice. Virus Genes 35: 663-671.

Li B, Fang L, Guo X, Gao J, Song T, Bi J, He K, Chen H, Xiao S. 2011. Epidemiology and evolutionary characteristics of the porcine reproductive and respiratory syndrome virus in China between 2006 and 2010. Journal of Clinical Microbiology 49:3175-3183.

Liu D, Zhou R, Zhang J, Zhou L, Jiang Q, Guo X, Ge X, Yang MH. 2011. Recombination analyses between two strains of porcine reproductive and respiratory syndrome virus in vivo. Virus Research 155: 473-486.

Liu J, Wei C, Lin Z, Fan J, Xia W, Dai A, Yang X. 2019. Recombination in lineage 1, 3, 5 and 8 of porcine reproductive and respiratory syndrome viruses in China. Infection Genetics and Evolution 68(5):119-126.

Liu J, Zhou X, Zhai J, Li B, Wei C, Dai A, Yang X, Luo M. 2017a. Emergence of a novel highly pathogenic porcine reproductive and respiratory syndrome virus in China. Transboundary and Emerging Diseases 64(6): 2059-2074.

Liu J, Zhou X, Zhai J, Li B, Wei C, Dai A, Yang X, Luo M. 2017b. Genetic diversity and evolutionary characteristics of type 2 porcine reproductive and respiratory syndrome virus in southeastern China from 2009 to 2014.Archives of Virology 162(9): 2603-2615.

Liu J, Zhou X, Zhai J, Wei C, Dai A, Yang X, Luo M. 2017c. Recombination in JXA1-R vaccine and NADC30-like strain of porcine reproductive and respiratory syndrome viruses. Veterinary Microbiology 204: 110-120.

Meulenberg JJ. 2000. PRRSV, the virus.Veterinary Research 31: 11-21.

Murtaugh MP, Stadejek T, Abrahante JE, Lam TT, Leung FC. 2010. The everexpanding diversity of porcine reproductive and respiratory syndrome virus. Virus Research 154 (1-2): $18-30$.

Neumann EJ, Kliebenstein JB, Johnson CD, Mabry JW, Bush EJ, Seitzinger AH, Green AL, Zimmerman JJ. 2005. Assessment of the economic impact of porcine reproductive and 
respiratory syndrome on swine production in the United States. Journal of the American Veterinary Medical Association 227: 385-392.

Shi M, Lam TT, Hon CC, Murtaugh MP, Davies PR, Hui RK, Li J, Wong LT, YipCW, Jiang JW, Leung FC. 2010. Phylogeny-based evolutionary, demographical, and geographical dissection of North American type 2 porcine reproductive and respiratory syndrome viruses. Journal of Virology 84: 8700-8711.

van Geelen AG, Anderson TK, Lager KM, Das PB, Otis NJ, Montiel NA, Miller LC, Kulshreshtha V, Buckley AC, Brockmeier SL, Zhang J, Gauger PC, Harmon KM, Faaberg KS. 2018. Porcine reproductive and respiratory disease virus: Evolution and recombination yields distinct ORF5 RFLP 1-7-4 viruses with individual pathogenicity. Virology 513:168-179.

Wang L, Wan B, Guo Z, Qiao S, Li R, Xie S, Chen XX, Zhang G. 2018. Genomic analysis of a recombinant NADC30-like porcine reproductive and respiratory syndrome virus in China. Virus Genes 54(1): 86-97.

Wang X, Dang R, Liu W, Yang Z, Du E, Zhang S. 2014. Antigenic characteristics of glycosylated protein 3 of highly pathogenic porcine reproductive and respiratory syndrome virus. Virus Research 189: 24-28.

Wei Z, Lin T, Sun L, Li Y, Wang X, Gao F, Liu R, Chen C, Tong G, Yuan S. 2012. N-linked glycosylation of GP5 of porcine reproductive and respiratory syndrome virus is critically important for virus replication in vivo. Journal of Virology 86(18): 9941-9951.

Wesley RD, Mengeling WL, Lager KM, Clouser DF, Landgraf JG, Frey ML. 1998. Differentiation of a porcine reproductive and respiratory syndrome virus vaccine strain from North American field strains by restriction fragment length polymorphism analysis of ORF5. Journal of Veterinary Diagnostic Investigation 10: 140-144.

Wissink EH, Kroese MV, Maneschijn-Bonsing JG, Meulenberg JJ, van Rijn PA, Rijsewijk FA, Rottier PJ. 2004. Significance of the oligosaccharides of the porcine reproductive and respiratory syndrome virus glycoproteins GP2a and GP5 for infectious virus production. 
340

341

342

343

344

345

346

347

348

Journal of General Virology 85: 3715-3723.

Wu WH, Fang Y, Rowland RR, Lawson SR, Christopher-Hennings J, Yoon KJ, NelsonEA. 2005. The $2 \mathrm{~b}$ protein as a minor structural component of PRRSV. Virus Research 114: 177181.

Zhang HL, Zhang WL, Xiang LR, Leng CL, Tian ZJ, Tang YD, Cai XH. 2018. Emergence of novel porcine reproductive and respiratory syndrome viruses (ORF5 RFLP 1-7-4 viruses) in China. Veterinary Microbiology 222:105-108.

Zhang Q, Jiang P, Song Z, Lv L, Li L, Bai J. 2016. Pathogenicity and antigenicity of a novel NADC30-like strain of porcine reproductive and respiratory syndrome virus emerged in China. Veterinary Microbiology 197: 93-101.

Zhao H, Han Q, Zhang L, Zhang Z, Wu Y, Shen H, Jiang P. 2017. Emergence of mosaic recombinant strains potentially associated with vaccine JXA1-R and predominant circulating strains of porcine reproductive and respiratory syndrome virus in different provinces of China. Virology Journal 14: 67.

Zhao K, Ye C, Chang XB, Jiang CG, Wang SJ, Cai XH, Tong GZ, Tian ZJ, Shi M, An TQ. 2015. Importation and recombination are responsible for the latest emergence of highly pathogenic porcine reproductive and respiratory syndrome virus in China. Journal of Virology 89: 10712-10716.

Zhou L, Kang R, Zhang Y, Ding M, Xie B, Tian Y, Wu X, Zuo L, Yang X, Wang H. 2018. Whole genome analysis of two novel type 2 porcine reproductive and respiratory syndrome viruses with complex genome recombination between lineage 8, 3, and 1 strains identified in Southwestern China. Viruses 10: pii: E328.

Zhou L, Yang H. 2010. Porcine reproductive and respiratory syndrome in China. Virus Research 154: $31-37$.

Zhou YJ, An TQ, He YX, Liu JX, Qiu HJ, Wang YF, Tong GZ. 2006. Antigenic structure analysis of glycosylated protein 3 of porcine reproductive and respiratory syndrome virus. 
366

367

368

369

370

371

372

373

374

375

376

377

378

379

380

381

382

383

384

385

Virus Research 118: 98-104.

Zhou YJ, Yua H, Tian ZJ, Liu JX, An TQ, Peng JM, Li GX, Jiang YF, Cai XH, Xue Q, Wang M, Wang YF, Tong GZ. 2009. Monoclonal antibodies and conserved antigenic epitopes in the $\mathrm{C}$ terminus of GP5 protein of the North American type porcine reproductive and respiratory syndrome virus. Veterinary Microbiology 138: 1-10.

\section{Figure legends}

Figure. 1 Phylogenetic tree based on the ORF5 genes (A) and full length (B) of the FJ0908 and reference viruses. Reliability of the tree was assessed by bootstrap analysis of 1000 replications. Our representative isolate FJ0908 were marked with the red triangle $(\boldsymbol{\Delta})$. Lineage 1 PRRSVs are divided into nine sublineages.

Figure. 2 Characterization of the supported recombinant events between FJL0908 and representative PRRSV lineages. (A) Similarity plot and bootscan analyses of FJ0908 by SimPlot. The y-axis indicates the percentage similarity between the parental sequences and the query sequence. Phylogenetic trees based on major parental regions (nt1-759 and nt 1301-15534) (B) and the minor parental region (nt760-1300) (C). The major parental group (1-7-4 viruses, reference strain IA/2014/NADC34) is shown in red, while the minor parental groups (reference strain ISU30) are shown in blue, respectively. 
Figure 1

Phylogenetic tree based on the ORF5 genes (A) and full length (B) of the FJ0908 and reference viruses.

Reliability of the tree was assessed by bootstrap analysis of 1000 replications. Our representative isolate FJ0908 were marked with the red triangle ( $\mathbf{\Lambda})$. Lineage 1 PRRSVs are divided into nine sublineages. 
A

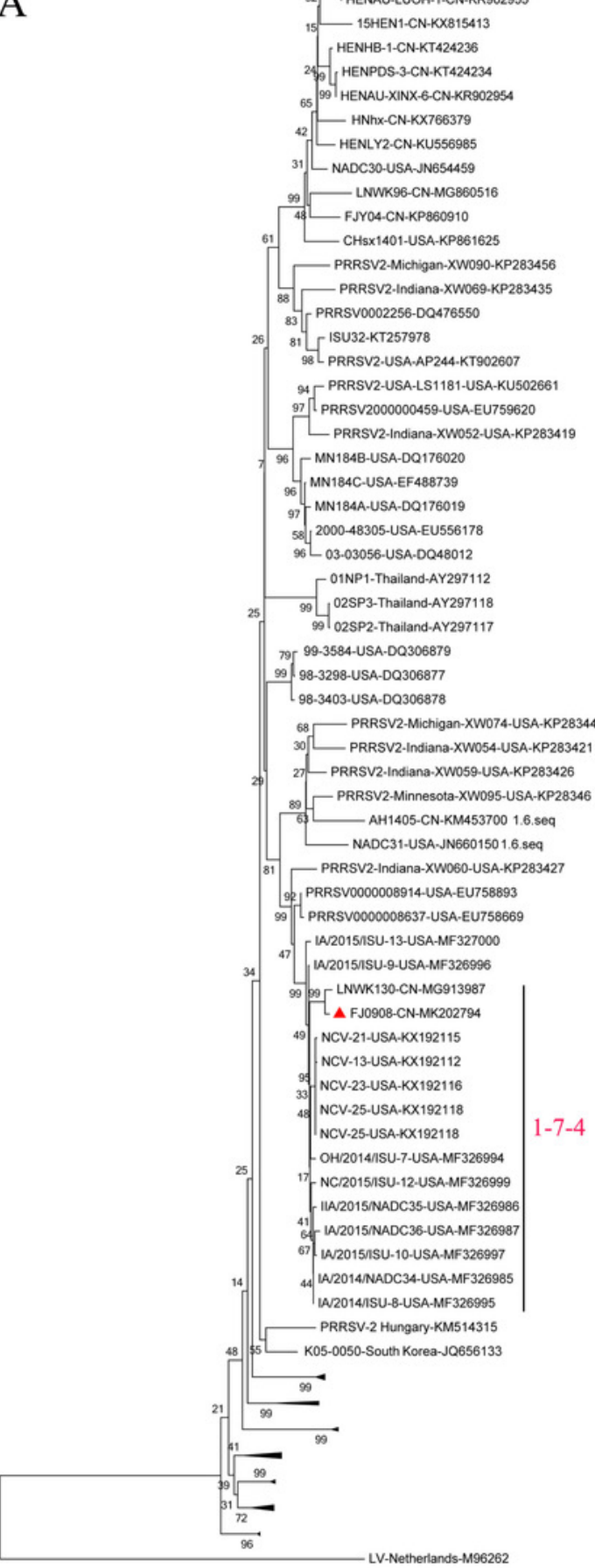

B

sublineage 1.8

sublineage 1.7

sublineage 1.9

B

sublineage 1.4

sublineage 1.1

sublineage 1.6

sublineage 1.5

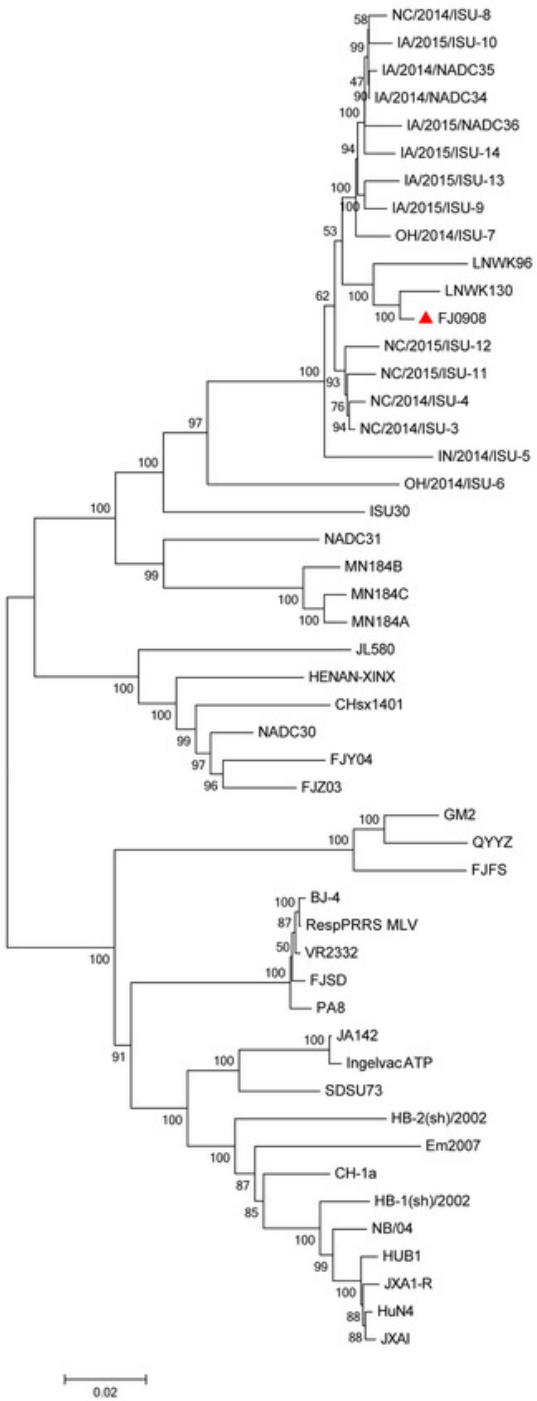

1-7-4-like

sublineage 1.2

| lineage 2

lineage 4

| lineage 3

lineage 9

sublineage 8.9

sublineage 8.7

| sublineage 5.1 


\section{Figure 2}

Characterization of the supported recombinant events between FJL0908 and representative PRRSV lineages.

(A) Similarity plot and bootscan analyses of FJ0908 by SimPlot. The y-axis indicates the percentage similarity between the parental sequences and the query sequence. Phylogenetic trees based on major parental regions (nt 1-760 and nt 1301-15534) (B) and the minor parental region (nt 760-1300) (C). The major parental group (1-7-4 viruses, reference strain IA/2014/NADC34) is shown in red, while the minor parental groups (reference strain ISU30) are shown in blue, respectively. 
A Simplot -Query FJ0908

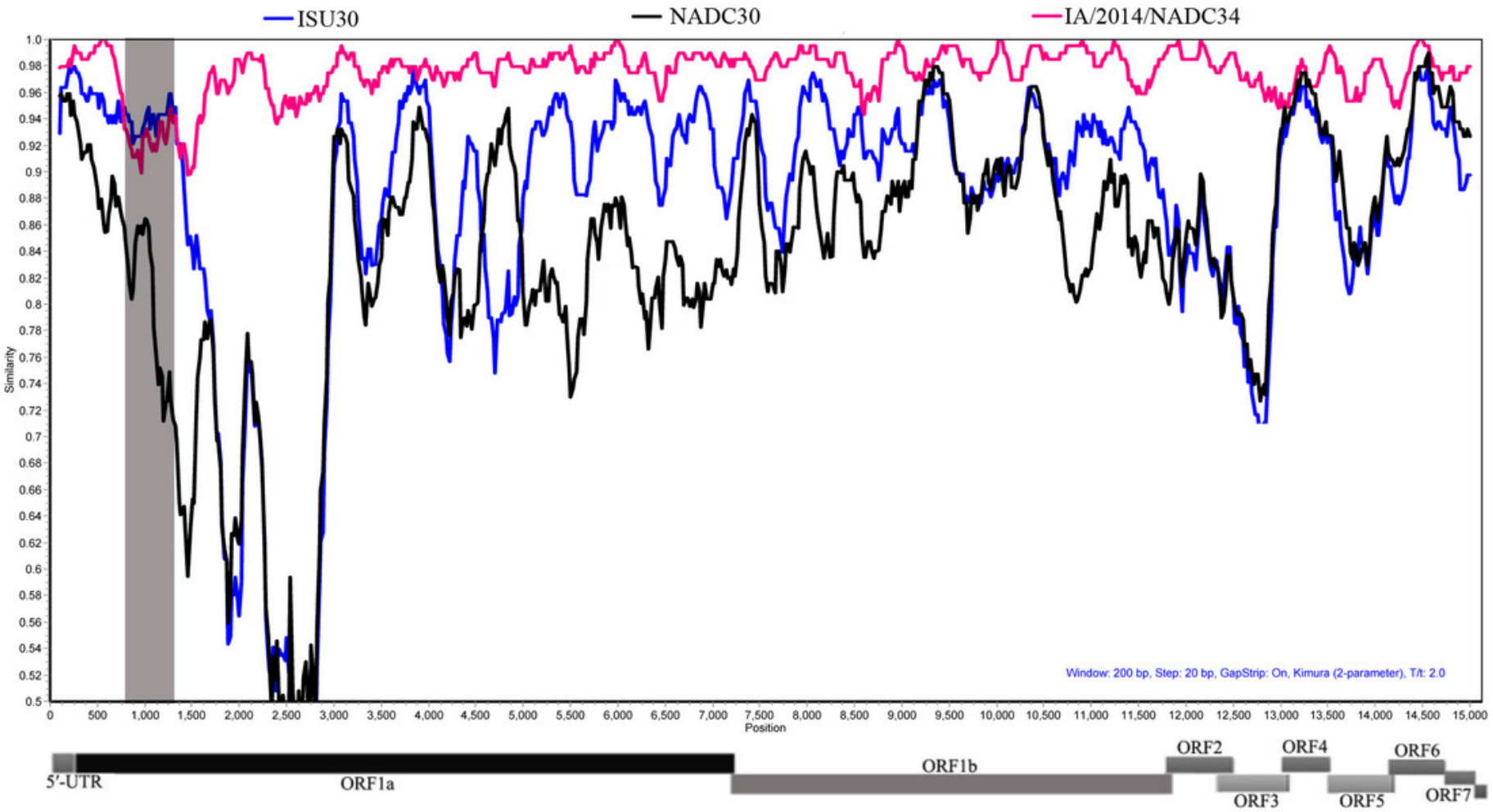

B

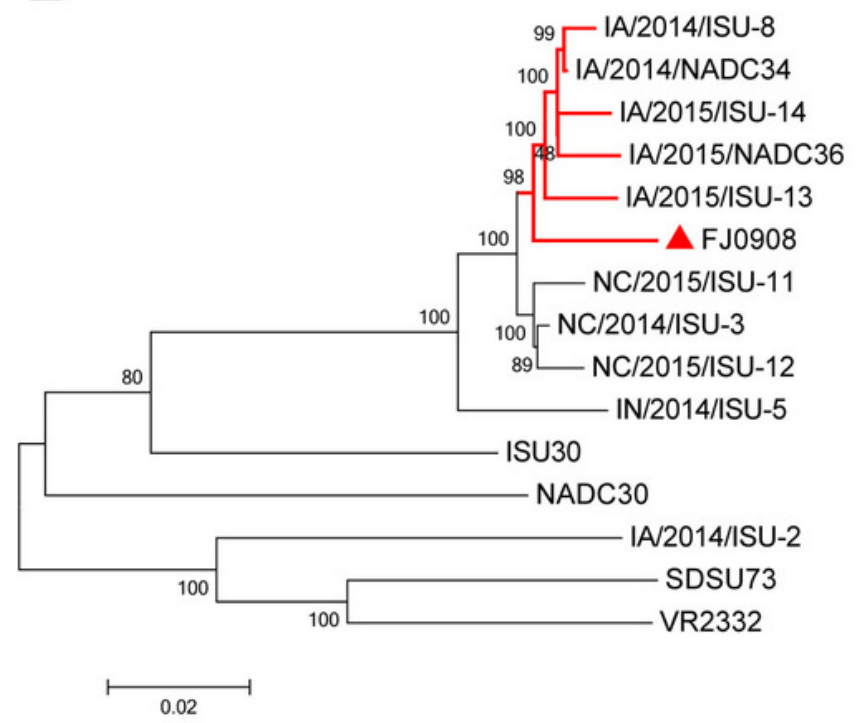

C

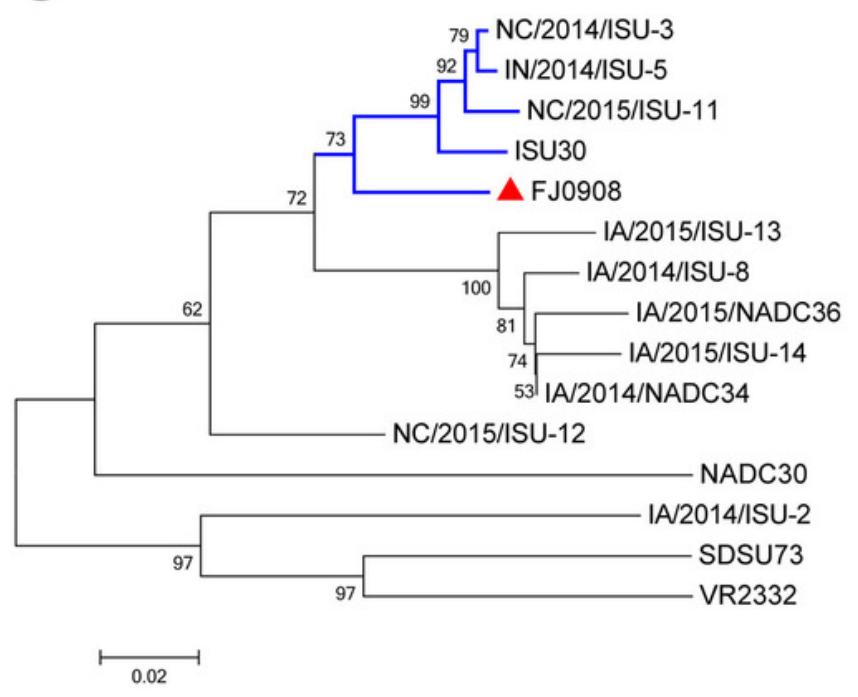




\section{Table $\mathbf{1}$ (on next page)}

PRRSV strains used in this study. 
1 Table 1 PRRSV strains used in this study.

\begin{tabular}{|c|c|c|c|c|c|c|c|}
\hline No. & Name & $\begin{array}{l}\text { GenBank } \\
\text { accession no. }\end{array}$ & Origin & No. & Name & $\begin{array}{l}\text { GenBank } \\
\text { accession no. }\end{array}$ & Origin \\
\hline 1 & FJ0908 & MK202794 & China & 29 & SDSU73 & JN654458 & U.S.A \\
\hline 2 & FJZ03 & KР860909 & China & 30 & NADC31 & JN660150 & U.S.A \\
\hline 3 & FJY04 & KP860910 & China & 31 & $\mathrm{NB} / 04$ & FJ536165 & China \\
\hline 4 & $\mathrm{CH}-1 \mathrm{a}$ & AY032626 & China & 32 & BJ-4 & AF331831 & China \\
\hline 5 & JA142 & AY424271 & U.S.A & 33 & MN184A & DQ176019 & U.S.A \\
\hline 6 & VR-2332 & U87392 & U.S.A & 34 & MN184B & DQ176020 & U.S.A \\
\hline 7 & JXA1-R & R FJ548855 & China & 35 & HENNAN-XINX & KF611905 & China \\
\hline 8 & JXA1 & EF112445 & China & 36 & NADC30 & JN654459 & U.S.A \\
\hline 9 & FJFS & KР998476 & China & 37 & JL580 & KR706343 & China \\
\hline 10 & PA8 & AF176348 & Canadia & 38 & CHsx1401 & KP861625 & China \\
\hline 11 & Em2007 & EU262603 & China & 39 & ISU30 & KT257977 & U.S.A \\
\hline 12 & GM2 & JN662424 & China & 40 & MN184C & EF488739 & U.S.A \\
\hline 13 & QYYZ & JQ308798 & China & 41 & NC/2014/ISU-3 & MF326990 & U.S.A \\
\hline 14 & HB-1(sh)/2002 & AY150312 & China & 42 & LV & M96262 & Netherlands \\
\hline 15 & HB-2(sh)/2002 & AY262352 & China & 43 & NC/2014/ISU-4 & MF326991 & U.S.A \\
\hline 16 & HUN4 & EF635006 & China & 44 & IN/2014/ISU-5 & MF326992 & U.S.A \\
\hline 17 & OH/2014/ISU-6 & MF326993 & U.S.A & 45 & Ingelvac ATP & DQ988080 & U.S.A \\
\hline 18 & NC/2015/ISU-11 & MF326998 & U.S.A & 46 & RespPRRS MLV & AF066183 & U.S.A \\
\hline 19. & IA/2015/ISU-13 & MF327000 & U.S.A & 47 & NC/2015/ISU-12 & MF326999 & U.S.A \\
\hline 20 & LNWK130 & MG913987 & China & 48 & IA/2015/NADC35 & MF326986 & U.S.A \\
\hline 21 & NCV-13 & KX192112 & U.S.A & 49 & IA/2015/NADC36 & MF326987 & U.S.A \\
\hline 22 & NCV-23 & KX192116 & U.S.A & 50 & IA/2015/ISU-10 & MF326997 & U.S.A \\
\hline 23 & NCV-25 & KX192118 & U.S.A & 51 & IA/2014/NADC34 & MF326985 & U.S.A \\
\hline 24 & OH/2014/ISU-7 & MF326994 & U.S.A & 52 & LNWK96-CN & MG860516 & China \\
\hline 25 & IA/2014/ISU-8 & MF326995 & U.S.A & 53 & NCV-21 & KX192115 & U.S.A \\
\hline 26 & IA/2015/NADC36 & MF326987 & U.S.A & 54 & IA/2015/ISU-9 & MF326996 & U.S.A \\
\hline 27 & FJSD & KP998474 & China & 55 & HUB1 & EF075945 & China \\
\hline 28 & IA/2014/ISU-2 & MF326989 & U.S.A & 56 & IA/2015/ISU-14 & MF327001 & U.S.A \\
\hline
\end{tabular}

2 
Table 2 (on next page)

Detailed comparison of the full-length genomes of FJ0908 to other PRRSV reference strains. 
1 Table2 Detailed comparison of the full-length genomes of FJ0908 to other PRRSV reference strains.

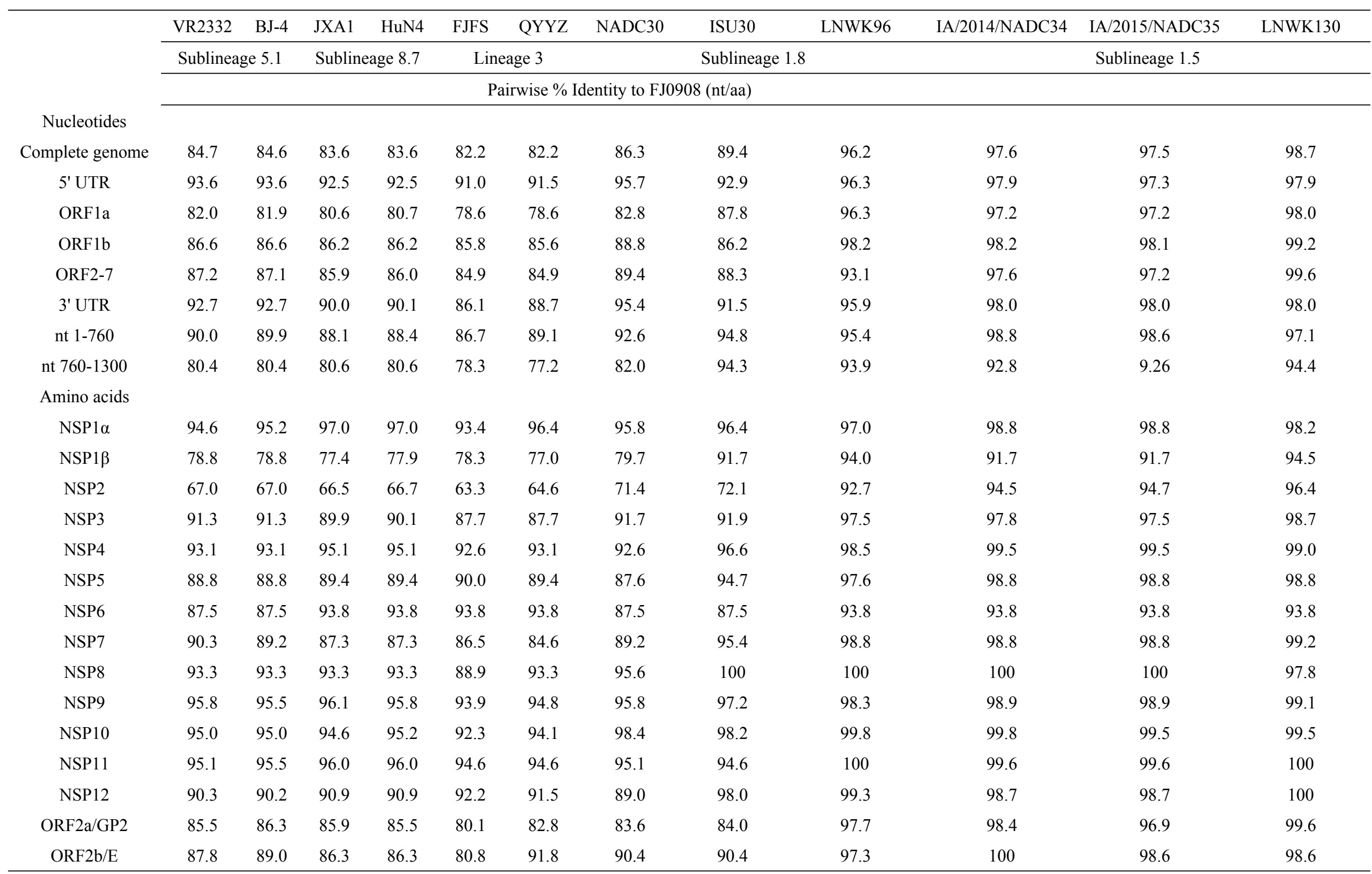




\begin{tabular}{cccccccccccc}
\hline ORF3/GP3 & 80.7 & 80.7 & 79.9 & 79.5 & 80.7 & 79.9 & 81.5 & 81.9 & 89.4 & 94.5 & 93.3 \\
ORF4/GP4 & 86.0 & 84.8 & 86.5 & 88.8 & 88.8 & 86.5 & 94.4 & 92.1 & 91.6 & 94.4 & 99.2 \\
ORF5/GP5 & 87.0 & 87.0 & 86.0 & 86.5 & 85.5 & 85.5 & 90.5 & 88.0 & 90.0 & 97.5 & 97.4 \\
ORF5a & 91.3 & 91.3 & 84.8 & 84.8 & 84.8 & 87.0 & 95.7 & 93.5 & 95.7 & 100 & 99.4 \\
ORF6/M & 95.4 & 94.8 & 93.7 & 93.7 & 95.4 & 94.8 & 94.8 & 93.7 & 95.4 & 97.7 & 98.5 \\
ORF7/N & 91.1 & 90.2 & 90.2 & 90.2 & 87.8 & 87.8 & 95.9 & 93.5 & 9.19 & 96.7 & 97.7 \\
\hline
\end{tabular}

2 